\title{
Energy utilization analysis and optimization of Correc- tive Insoles manufactured by 3D Printing
}

\author{
M.J. Kirby ${ }^{1}$, R.L. Johnson ${ }^{1}$, A. Rees ${ }^{1}$, C.A.Griffiths ${ }^{1}$ \\ ${ }^{1}$ College of Engineering, Swansea University, UK
}

\begin{abstract}
The foot orthotic insole market is forecast to surpass a value of 3.6 billion USD by 2021 . This vast industry continues to rely on foam milling and other subtractive methods of manufacturing, which have proven to be wasteful and inefficient. Leaps in digital manufacturing have enabled the technology to enter a plethora of industries, with the promise of increased customization accompanied with reduced waste generation. Despite boasting these valuable traits, the explosive proliferation of 3D printing in conjunction with mounting pressure to incorporate sustainable practices, means that research must be focused on maximizing the material and energy efficiency of the technology. This paper employs a Design of Experiments (DoE) approach for the optimization of two prefabricated insoles, adjusting percentage infill and layer height to obtain data regarding the effects of these parameters on print time, filament usage volume, and energy consumption. Key conclusions formed from the study were that infill density is the dominant factor effecting material consumption and power usage, whereas layer height has the greatest influence on production time. The data presented in this study has the potential to aid not only in the development of mass producible additive manufactured (AM) insoles, but also to advance the understanding of the environmental impact of AM technologies.
\end{abstract}

Keywords: 3D printing; additive manufacturing; green manufacturing

\section{$1 \quad$ Introduction}

\subsection{Anatomical insoles}

Anatomical insoles are functional additions to any shoe, used primarily to apply a force on the body to counteract the effects of a biomechanical disorder; for example flat feet, which can be hereditary, or simply caused by a foot or ankle injury [1]. Deformities such as this often have a transitive effect on the rest of the supporting joints above the foot/ankle, also known as the kinetic chain, manifesting as symptomatic conditions such as shin splints, Achilles tendonitis, and plantar fasciitis. Insoles secondary beneficial aspect is that they provide improved cushioning when compared to the original factory shoe insole, alleviating some of the pain associated with the impact caused by walking [2].

Traditional methods of manufacturing insoles, like Computer Numerical Control (CNC) foam milling, continue to be the industry standard, despite the rapid proliferation of three-dimensional (3D) printing; a substantially more customizable, less wasteful and in many ways, cheaper alternative [3,4]. One of the reasons for the delayed 
uptake of $3 \mathrm{D}$ printing is that suitable materials with comparable mechanical properties to the Ethylene-vinyl acetate (EVA) foam employed in traditional milling processes are not yet readily available to the extent that would be required for the mass production of insoles. A pertinent study conducted by Salles and Gyi in 2013 compared the functionality of traditionally manufactured insoles with additive manufacturing (AM) insoles [5]. One of their conclusions was that of the limited array of materials available for $\mathrm{AM}$, none displayed the cushioning properties required to rival their machined counterparts.

The relevance of reducing the underpinning manufacturing energy and material usage in the orthotic insole industry can be evidenced in the expansion of the global foot orthotic insole market value increased from approximately 2.7 billion USD in 2015 to over 3.1 billion USD in 2018 [6]. This figure is forecast to grow to almost 3.7 billion USD by 2021 [6].

The aim of this study, through the employment of a Design of Experiments (DoE) approach, is to quantify the energy and material usage for the manufacture of anatomical insoles through 3D printing.

\section{State of the Art}

\subsection{Orthotic Insole Manufacturing Techniques and Comparison}

While a diverse range of natural and synthetic materials are used in the manufacture of foot orthoses, they are often categorized into three main types; soft, semi-rigid, and rigid. The recent development of advanced materials, such as carbon graphite fibre and silicon, have allowed the fabrication of orthosis devices to progress at a rapid rate, as well as the corresponding manufacturing techniques.

\subsection{D printing insoles}

With use of customization and control as the experimental conditions, Salles et al., produced AM manufactured insoles to be tested on study participants for 3 months. In particular, participants were blindly assigned either a personalized 'glove fit' insole mimicking the geometry of their feet, while the remaining participants were given control insoles that copied the geometry of the test shoe's factory insole. The research found that the 'glove fit' insoles considerably improved the comfort in the heel area and general fit of the participants during running, when compared to the control article. This was done by minimizing ankle dorsiflexion, thus reducing the peak pressure at the point of impact with the ground. However, the study observed that discomfort under the arch persisted throughout the test period. The research concluded that with the advancement of 3D printing technology, AM manufactured personalized insoles can be used as an affordable and beneficial addition to footwear [5].

In contrast, Davia-Aracil et al., conducted research into a developing an innovative programme focused entirely on simplifying the design and production of AM manufactured personalized insoles, where users could specify desired support dimensions. 
Through this, the researchers concluded that although the footwear industry could benefit hugely from additive manufacturing, it currently lacks the CAD tools and experience to exploit the technological advancements of 3D printing [7].

\subsection{Sustainable AM techniques}

The quality and functionality of components have been the predominant focus of research into the optimization of manufacturing processes over recent decades, while the energy efficiency and subsequent environmental impact of said processes have been somewhat overlooked. However, as the emphasis on sustainable production grows, with the creation of laws and standards such as ISO 14000, Researchers and Engineers are being put under increasing pressure to drastically improve environmental management [8]. By incorporating an additional design stage into the Life Cycle Assessment (LCA) of a product with the sole purpose of reducing AM processes' environment impact, Tang et al., were able to compare the $\mathrm{CO}_{2}$ produced by $\mathrm{CNC}$ and $\mathrm{AM}$ processes. The research showed that, for the same product, AM techniques produce considerably less $\mathrm{CO}_{2}$ and have lower energy consumption than $\mathrm{CNC}$ milling [9].

In a study by Peng it was concluded that $3 \mathrm{D}$ printing can be optimized through multiobjective process optimization with an emphasis on 'green' performance. In particular, the research found that through the use of model-based energy evaluation built on initial CAD models, optimal energy utilization can only be achieved during all stages of manufacture [10].

Griffiths et al., establishing a Design of Experiments (DoE) approach to optimizing additive manufacturing techniques. In particular, the investigation studied the efficacy of inputting environmentally conscious build parameters into a FDM process to analyze the response. The research concludes that layer height is the most significant parameter with regards to energy consumption and production time [11]. Additional research supporting this concept was introduced by Mognol et al., who focused on determining a set of constraints with the purpose of reducing the electrical energy consumption of three AM processes [12].

The aim of this study, through the employment of a Design of Experiments (DoE) approach, is to quantify the energy and material usage for the manufacture of anatomical insoles through 3D printing.

\section{$3 \quad$ Methodology}

\subsection{Materials and Equipment}

To establish the optimal build parameter settings, percentage infill and layer height will be varied to minimize the energy and material consumption of the FDM manufacturing process. To ensure an informed assessment of the build parameter influence is achieved, two distinct prefabricated insoles geometries have been selected for testing. In particular, a $7 \mathrm{~mm}$ Heel Lift and a $3 / 4$ length Heel Cup Arch Support (Figure 1). 

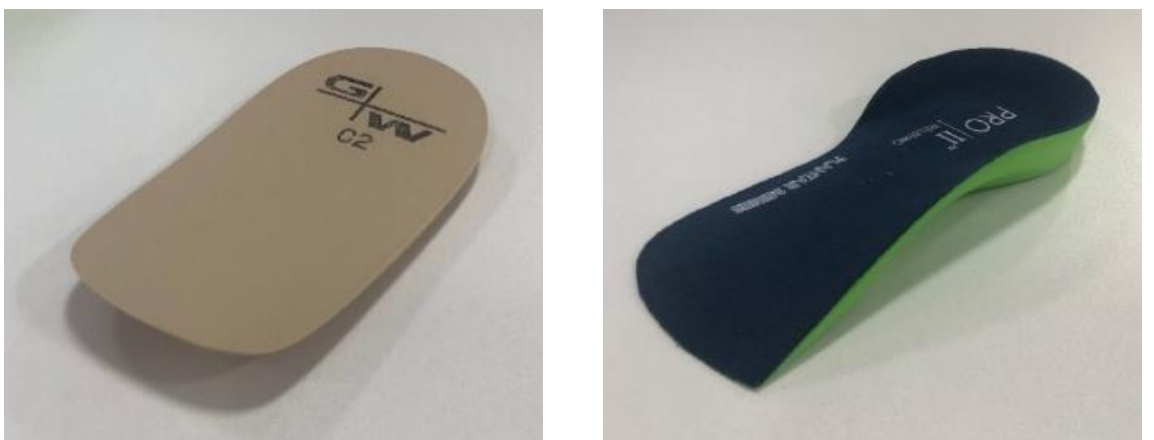

Figure 1 - Heel Lift (Left) and Arch Support (Right)

The chosen material for the tests, Polylactic acid (PLA), is a biodegradable polymer manufactured from plants including sugarcane and corn, and is one of the most frequently used materials in printing technologies.

The AM equipment used was an Ultimaker 2+ (Figure 2); a printer that uses Fused Filament Fabrication (FFF) technology. The printer used $2.85 \mathrm{~mm}$ diameter PLA filament. The remaining key technical characteristics of the Ultimaker $2+$ are displayed in Table 2.

To facilitate the measurement of power consumption throughout each of the tests, a power usage meter with the ability to measure cumulative power intake was required. The power usage meter chosen, pictured in Figure 3, measured the total energy usage

Table 2. Ultimaker $2+$ specification [13]

\begin{tabular}{|l|l|}
\hline Characteristic & Value \\
\hline Build Volume $\left(\mathrm{mm}^{3}\right)$ & $223 \times 223 \times 205$ \\
\hline Build Speed $\left(\mathrm{mm}^{3} / \mathrm{s}\right)$ & $<16$ \\
\hline Nozzle Diameter $(\mathrm{mm})$ & 0.4 \\
\hline XYZ Resolution $(\mu \mathrm{m})$ & $12.5(x), 12.5(y), 5(z)$ \\
\hline
\end{tabular}
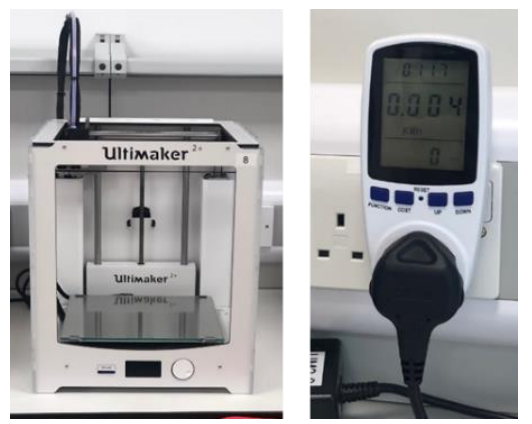

Figure 2 - Ultimaker 2+ (left) and Power Usage Meter (Right) 


\subsection{Design of Experiments}

Through a Design of Experiments (DoE) the processing variables percentage infill and layer height were adjusted. In addition, a control experiment (A0 \& H0) was conducted whereby an infill of $100 \%, 0.15 \mathrm{~mm}$ layer height, $0.7 \mathrm{~mm}$ shell thickness, a shell number of 2 , and a print speed of $50 \mathrm{~mm} / \mathrm{s}$ were used. Table 3 displays the DoE array of combinations used within the experiments. Also, a triangular infill pattern was used throughout all tests due to its high ratio of perpendicular compressive strength to print time, when compared to other infill patterns $[14,15]$.

Table 1 - Design of Experiments for Each Insole

\begin{tabular}{ccccc}
\hline Case ID & \% Infill & $\begin{array}{c}\text { \% Infill Plot } \\
\text { Key }\end{array}$ & $\begin{array}{c}\text { Layer Height } \\
(\mathbf{m m})\end{array}$ & $\begin{array}{c}\text { Layer Height } \\
\text { Plot Key }\end{array}$ \\
\hline A0 & $\mathbf{1 0 0}$ & N/A & $\mathbf{0 . 1 5}$ & N/A \\
A1 & 40 & 1 & 0.15 & -1 \\
A2 & 40 & 1 & 0.2 & 1 \\
A3 & 20 & -1 & 0.15 & -1 \\
A4 & 20 & -1 & 0.2 & 1 \\
H0 & $\mathbf{1 0 0}$ & N/A & $\mathbf{0 . 1 5}$ & N/A \\
H1 & 60 & 1 & 0.15 & 1 \\
H2 & 60 & 1 & 0.1 & -1 \\
H3 & 10 & -1 & 0.15 & 1 \\
H4 & 10 & -1 & 0.1 & -1 \\
\hline
\end{tabular}

Table 4 - Experimental Data for All Cases

\begin{tabular}{cccccc}
\hline $\begin{array}{c}\text { Case } \\
\text { ID }\end{array}$ & \% Infill & $\begin{array}{c}\text { Layer Height } \\
(\mathbf{m m})\end{array}$ & Printing time $(\mathbf{d}: \mathbf{h}: \mathbf{m})$ & Filament usage $\left(\mathbf{m}^{\mathbf{3}}\right)$ & $\begin{array}{c}\text { Power usage } \\
(\mathbf{k W h})\end{array}$ \\
\hline A0 & $\mathbf{1 0 0}$ & $\mathbf{0 . 1 5}$ & $\mathbf{0 1 : 0 6 : 5 5}$ & $\mathbf{0 . 0 0 0 1 1 9 8 0 5}$ & $\mathbf{3 . 7 6 5}$ \\
A1 & 40 & 0.15 & $00: 10: 10$ & 0.000063411 & 1.285 \\
A2 & 40 & 0.2 & $00: 07: 15$ & 0.000061816 & 1.216 \\
A3 & 20 & 0.15 & $00: 08: 03$ & 0.000044337 & 1.027 \\
A4 & 20 & 0.2 & $00: 05: 37$ & 0.000042232 & 0.706 \\
H0 & $\mathbf{1 0 0}$ & $\mathbf{0 . 1 5}$ & $\mathbf{0 0 : 0 2 : 5 4}$ & $\mathbf{0 . 0 0 0 0 1 1 1 6 4}$ & $\mathbf{0 . 3 9 4}$ \\
H1 & 60 & 0.15 & $00: 01: 53$ & 0.000010079 & 0.246 \\
H2 & 60 & 0.1 & $00: 02: 39$ & 0.000009952 & 0.340 \\
H3 & 10 & 0.15 & $00: 01: 36$ & 0.000007464 & 0.198 \\
H4 & 10 & 0.1 & $00: 02: 11$ & 0.000007145 & 0.270 \\
\hline
\end{tabular}




\section{$4 \quad$ Results \& Discussion}

\subsection{Printing Time}

For the Heel Lift component, the experimental results suggest that percentage infill and layer height have a significant effect on the overall print time. The Main Effects plots displayed in Figures 3 and 4 highlight that, for both specimens, a greater layer height corresponds to a decrease in print time. This is supported by a $27.83 \%$ average reduction in production time for the Heel Lift between the two tested levels. This can be attributed to a reduction in the quantity of layers required to produce an equivalent structure.

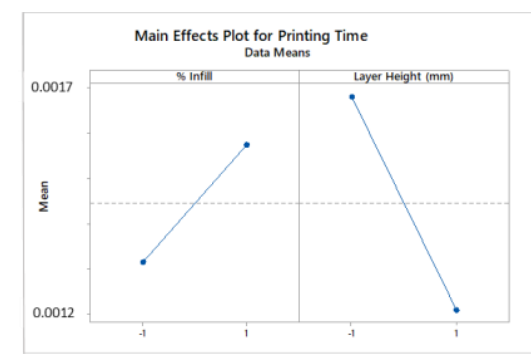

Figure 3 - Heel Lift

A very similar outcome can be observed from the Arch Support data involving print time. This is referring to layer height being the critical factor, exhibiting a $29.49 \%$ drop in print time between $0.15 \mathrm{~mm}$ layer thickness and $0.2 \mathrm{~mm}$. Varying the percentage infill had a sizeable yet reduced influence of $21.23 \%$ and $16.33 \%$ for the Arch Support and Heel Lift respectively. Since nozzle diameter and print speed were kept constant throughout the experiment, it can be deduced that the overall increase in density of the printed structure caused by increased infill resulted in a greater amount of PLA required, and consequently lengthened the production time (Figure 4).

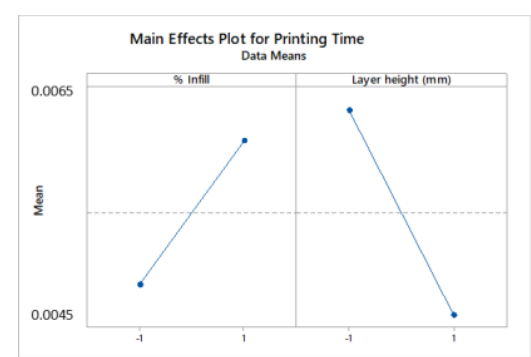

Figure 4 - Arch Support 


\subsection{Filament Usage}

The specific factor causing the most significant change in filament usage volume in both specimens is percentage infill, by a substantial margin. This is displayed clearly in Figures 5 and 6, and has been calculated to have caused a $27.08 \%$ and $30.88 \%$ reduction in the Heel Lift and Arch Support respectively. Hence a decrease in the percentage infill will result in a decrease in material consumption, but will also have damaging effects on the mechanical properties of the printed part.

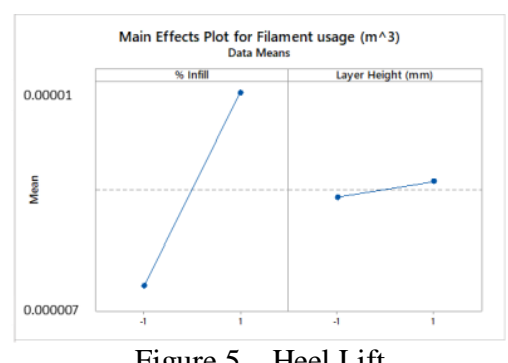

Figure 5 - Heel Lift

However, modifying the layer height evidently has a negligible effect on material usage, with the variation from $0.1 \mathrm{~mm}$ to $0.15 \mathrm{~mm}$ resulting in a $2.77 \%$ increase for the Heel Lift, whereas the change from $0.15 \mathrm{~mm}$ to $0.2 \mathrm{~mm}$ produced a $3.64 \%$ decrease in filament consumption for the Arch Support. This can be attributed to the fact that increasing layer height causes filament extrusion rate to increase, up to the nozzle diameter.

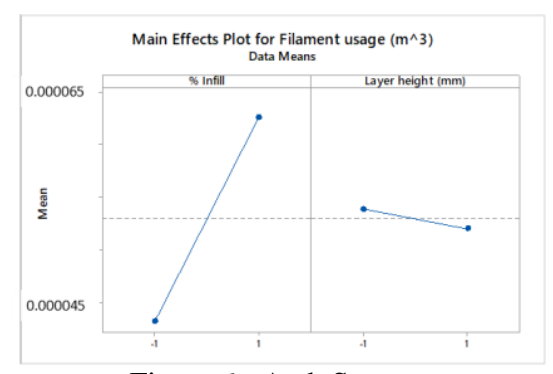

Figure 6 - Arch Support

Although it has a minor influence, and would require testing in isolation to provide a clearer outcome, one aspect of the data regarding layer height warrants mentioning. Figures 9 and 10 show that for both insoles, a layer thickness of $0.15 \mathrm{~mm}$ increased the filament usage volume. As a result of this, the critical settings for reducing filament usage are $10 \%$ infill and $0.1 \mathrm{~mm}$ layer height for the Heel Lift, while $20 \%$ infill along with a $0.2 \mathrm{~mm}$ layer thickness are recommended for the Arch Support. 


\subsection{Power Usage}

With respect to energy consumption, inconsistencies are present in the effects of both build parameters. The key factor for the Heel Lift is layer height (Figure 7) with a reduction of $27.16 \%$. This is compared to a $31.01 \%$ drop due to percentage infill having a greater impact on the Arch Support (Figure 8). As stated in section 4.1, increasing the layer height cuts the print time due to a reduction in the number of layers required to reach a specified part height. A lower print time causes a subsequent decline in energy consumption, and supports the conclusion presented by Griffiths et al., [11]. The impact of percentage infill on the Arch Support indicates that the increased number of direction changes as a result of a higher infill density causes a simultaneous increase in energy consumption.

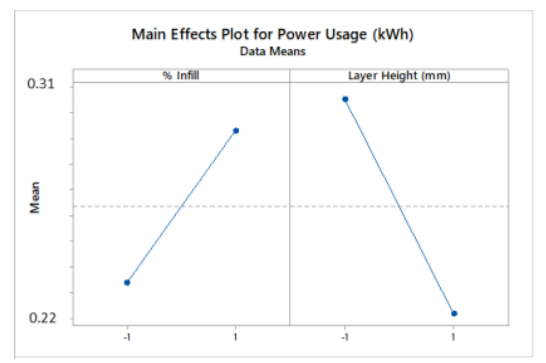

Figure 7 - Heel Lift

Focusing on the Arch Support, considerable disparities were observed in the effect of layer height. At $40 \%$ infill, it experienced only a $5.37 \%$ reduction in energy consumption between a $0.15 \mathrm{~mm}$ and $0.2 \mathrm{~mm}$ layer height (Table 4). However, for the same change in layer height but at $20 \%$ infill, this difference was $31.26 \%$. Corresponding values of $27.65 \%$ and $26.67 \%$ were obtained for the Heel Lift; a difference of only $0.98 \%$. This is compared to the $25.89 \%$ difference for the Arch Support, displaying the impact of interacting parameters on power consumption, irrespective of the singular build parameters used.

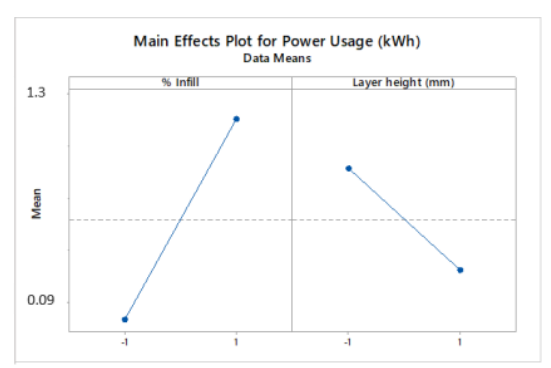

Figure 8 - Arch Support

For both samples, percentage infill is optimized with the lower value, and works in conjunction with a higher layer height to minimize energy usage. The optimal settings for each part, of those tested, are as follows; $10 \%$ infill and $0.15 \mathrm{~mm}$ layer height for the 
Heel Lift, while the Arch Support would benefit from a $20 \%$ infill and $0.2 \mathrm{~mm}$ layer height.

\section{Conclusion}

The key objectives of this paper is to investigate the optimal settings of build parameters in the manufacture of anatomical insoles by 3D printing in a bid to improve energy and material efficiency. Through conducting a DoE approach the following conclusions can be drawn:

- When printing the Arch Support a considerable resultant difference was observed in energy usage when utilizing identical layer heights when modifying the level of infill density. This leads to a conclusion that interactions are present between the build parameters.

- Layer height proved to be the most influential factor with respect to print time. A higher layer thickness results in less layers, or greater z-axis increments required to achieve an equivalent part thickness, thus reducing the production time.

- When optimizing for material consumption the Heel Lift must be manufactured with a $0.1 \mathrm{~mm}$ layer height at $10 \%$ infill, while the Arch Support should use $20 \%$ infill with a $0.2 \mathrm{~mm}$ layer thickness.

- For optimal energy consumption the Heel Lift requires a $10 \%$ infill with a $0.15 \mathrm{~mm}$ layer height. The Arch Support requires a $20 \%$ infill and a layer height of $0.2 \mathrm{~mm}$.

\section{Future Consideration}

Performing tensile tests on the printed specimens could be used to further expand the corrective aspect of the insoles whereby additional functionality could be gained by varying the resultant mechanical properties to align with the varying biomechanical requirements of the end user.

\section{$7 \quad$ Acknowledgement}

The author would like to acknowledge the financial support of the College of Engineering, Swansea University to facilitate conference funding.

\section{$8 \quad$ References}

[1] Crabtree P, Dhokia V, Newman S, Ansell M. Manufacturing methodology for personalised symptom-specific sports insoles. Robotics and Computer-Integrated Manufacturing. 2009;25(6):972-979. 
[2] Tuff S. Do You Really Need an $\$ 800$ Custom Insole? [Internet]. Nytimes.com. 2019 [cited 10 December 2018]. Available from: https://www.nytimes.com/2006/06/22/fashion/thursdaystyles/22Fitness.html

[3] Lipson H, Kurman M. Fabricated: The New World of 3D Printing. Indianapolis, Ind.: J. Wiley \& Sons; 2013.

[4] Gebler M, Schoot Uiterkamp A, Visser C. A global sustainability perspective on 3D printing technologies. Energy Policy. 2014;74:158-167.

[5] Salles A, Gyi D. An evaluation of personalised insoles developed using additive manufacturing. Journal of Sports Sciences. 2013;31(4):442-450.

[6] Foot orthotic insoles market size globally 2015-2021 | Statistic [Internet]. Statista. 2019 [cited 13 February 2019]. Available from: https://www.statista.com/statistics/888548/foot-orthotic-insoles-market-size-global/

[7] Davia-Aracil M, Hinojo-Pérez J, Jimeno-Morenilla A, Mora-Mora H. 3D printing of functional anatomical insoles. Computers in Industry. 2018;95:38-53.

[8] International Standards Organization (ISO). ISO 14000: Environmental Management. Geneva: ISO; 2015.

[9] Tang Y, Mak K, Zhao Y. A framework to reduce product environmental impact through design optimization for additive manufacturing. Journal of Cleaner Production. 2016;137:1560-1572.

[10] Peng T. Analysis of Energy Utilization in 3D Printing Processes. Procedia CIRP. 2016;40:62-67.

[11] Griffiths C, Howarth J, De Almeida-Rowbotham G, Rees A, Kerton R. A design of experiments approach for the optimisation of energy and waste during the production of parts manufactured by 3D printing. Journal of Cleaner Production. 2016;139:74-85. [12] Mognol P, Lepicart D, Perry N. Rapid prototyping: energy and environment in the spotlight. Rapid Prototyping Journal. 2006;12(1):26-34.

[13] Ultimaker 2+ Specification sheet [Internet]. Ultimaker; 2013 [cited 13 February 2019]. Available from: https://ultimaker.com/file/download/productgroup/Ultimaker\%202+\%20and\%20Ultimaker\%202\%20Extended+\%20specification\%20sheet.pdf/5b924f26322d6.pdf

[14] Dudescu C, Racz L. Effects of Raster Orientation, Infill Rate and Infill Pattern on the Mechanical Properties of 3D Printed Materials. ACTA Universitatis Cibiniensis. 2017;69(1):23-30.

[15] TESTING 3D printed INFILL PATTERNS for their STRENGTH [Internet]. YouTube. 2018 [cited 20 March 2019]. Available from: https://www.youtube.com/watch?v=upELIOHmzHc

[16] Kuznetsov V, Solonin A, Tavitov A, Urzhumtsev O, Vakulik A. Increasing of Strength of FDM (FFF) 3D Printed Parts by Influencing on Temperature-Related Parameters < strong > </strong > of the Process. 2018;

[17] Gunaydin K. The Effect of Layer Thickness to the Tensile Stress: Experimental Studies. International Congress on 3D Printing (Additive Manufacturing) Technologies and Digital Industry. Istanbul; 2018. 